\title{
Uma abordagem integrada sobre ontologias: Ciência da Informação, Ciência da Computação e Filosofia
}

Maurício Barcellos Almeida

Doutor em Ciência da Informação pela Escola de Ciência da Informação da UFMG.Pós-Doutor pela State University of New York at Buffalo. Professor do Programa de Pós Graduação da Escola de Ciência da Informação da UFMG.

http://dx.doi.org/10.1590/1981-5344/1736

Ontologias têm sido propostas como uma alternativa para a criação de representações da realidade adequadas a computadores. Ao buscar pelo termo "ontologia" em um mecanismo de busca, abordagens bastante diferenciadas são encontradas. De fato, o termo "ontologia" tem sido empregado na Filosofia, Ciência da Computação e Ciência da Informação com diferentes particularidades. Para fazer bom uso do que as teorias ontológicas têm a oferecer é preciso entender o que tais teorias abordam e quais as suas origens. Esse artigo busca esclarecer o que significa estudar ontologias e quais as conexões o assunto pode fomentar entre diferentes campos de pesquisa. Inicia-se revisitando o sentido do termo no campo de pesquisa onde ele foi criado, a Filosofia, para em seguida buscar entender o seu uso atual em outros campos. Propõe-se então que o estudo de ontologias é um assunto genuíno e relevante de pesquisa em Ciência da Informação. Finalmente, conclui-se oferecendo uma visão sobre as oportunidades de pesquisa interdisciplinar no assunto.

Palavras-chave: Ontologia, Ciência da Informação

\section{An unified approach to ontologies: Information Science, Computer Science and Philosophy}

Ontologies have been proposed as an alternative for creating representations of reality suitable for computers. In looking for "ontology" in a search engine, one can find many different approaches. Indeed, the term "ontology" is 
employed within Philosophy, Computer Science and Information Science with different meanings. In order to take advantage of what ontology theories have to offer, one should understand what they address and where they come from. This paper aims to clarify what means to study ontologies and what connections this subject fosters among different research fields. We begin by revisiting the meaning of the term ontology in its original field, Philosophy, to reach its current use in other research fields. We advocate that ontology is a genuine and relevant subject of research in Information Science. Finally, we conclude by offering our view of the opportunities for interdisciplinary research.

Key-words: Ontology, Information Science

Recebido em 27.03.2013 Aceito em 06.05.2013

\section{Introdução}

Dentre as diversas técnicas disponíveis para organização da informação e do conhecimento, a aplicação de ontologias tem sido recebido cada vez mais atenção. Ontologia é um assunto que tem sido estudado em diferentes campos de pesquisa - Filosofia, Ciência da Computação e Ciência da Informação - e no âmbito de vários domínios do conhecimento - Medicina, Biologia, Direito e Geografia. Apesar do uso e difusão do termo, não é uma tarefa simples entender o que significa "ontologia" e se estudar o assunto pode ser útil para a pesquisa em Ciência da Informação.

$\mathrm{Na}$ Filosofia, ontologia é então um ramo da Metafísica que diz respeito às quais categorias de entidades existem e estão relacionadas (LOWE, 2007). Em Ciência da Computação, ontologias são aplicadas à modelagem, tanto em sistemas baseados em bancos de dados quanto em sistemas de representação do conhecimento.

Em Ciência da Informação, abordagens ontológicas têm sido adotadas, mesmo que sob diferentes denominações (VICKERY, 1997). Alguns autores chegam mesmo a afirmar que ontologia é um novo nome para coisas já conhecidas (CURRÁS, 2003; GILCHRIST, 2003; SOERGUEL, 1999). Ao avaliar a emergência do termo ontologia em Ciência da Computação, Vickery (1997, p. 285) conclui que "os problemas com os quais os cientistas da informação vêm lutando há muito tempo são enfrentados agora pela comunidade de engenheiros do conhecimento."

Uma busca em publicações nacionais e internacionais de Ciência da Informação retorna poucas referências com uma explicação abrangente sobre o assunto, como por exemplo Soerguel (1997), Fonseca (2007), Schiessl e Bräscher (2011), Campos, Campos e Medeiros (2011). Esse artigo aborda ontologias sobre um ponto de vista interdisciplinar. Para tal, 
revisita-se o significado do termo "ontologia" em seu campo de pesquisa original, a Filosofia; além de seu uso em campos de pesquisa aplicados, tais como Ciência da Computação e Ciência da Informação. Espera-se assim identificar a conexão entre os diferentes usos do termo e encontrar os princípios comuns que unem as diversas visões. O que se argumenta aqui é que o assunto "ontologia" não está limitado a Ciência da Computação, como pode parecer executando uma simples pesquisa em um mecanismo de busca (RITTGEN, 2012). Ao invés disso, advoga-se que os princípios ontológicos são um assunto relevante de pesquisa em Ciência da Informação.

No restante do presente artigo adota-se a seguinte notação (GUARINO; GIARETTA, 1995): "ontologia", com letra inicial minúscula, refere-se a um artefato; "Ontologia", com letra inicial maiúscula, refere-se a abordagem filosófica. Utiliza-se também a expressão "princípios ontológicos" para se referir à fundamentos filosóficos, como por exemplo, teoria todo-parte, tipos e instanciação, identidade, dependência e unidade.

O restante do presente artigo é organizado como segue: a segunda seção apresenta algumas das principais abordagens filosóficas; a terceira seção descreve como termo ontologia é usado em outros campos científicos; a quarta seção discute questões referentes a sistemas de categorias; finalmente, a seção cinco oferece conclusões sobre a importância dos princípios ontológicos para a Ciência da Informação.

\section{0 que é Ontologia?}

Existe consenso de que o estudo da Ontologia diz respeito aos tipos de coisas que existem. Nesse contexto, "tipo" quer dizer "categoria", um termo que foi usado ainda por Aristóteles para discutir que declarações sobre uma entidade (ACKRILL, 1963). De fato, uma teoria das categorias é o mais importante tópico do estudo da Ontologia. Tais teorias especificam sistemas de categorias estruturados em níveis hierárquicos, em geral, na forma de uma árvore invertida na qual a categoria de mais alto nível é nomeada "entidade". Qualquer coisa pode ser descrita como uma entidade de algum tipo, mas qual os próximos níveis de categorização são questão para discussão.

Em função de sua influência nas propostas contemporâneas, pelos menos três sistemas de categorias devem ser analisados: o aristotélico, o kantiano e o husserliano (THOMASON, 2009). No restante da presente seção, descreve-se brevemente as principais características de cada um desses sistemas.

\subsection{A Ontologia em Aristóteles}

Aristóteles parece ter sido o primeiro filósofo a usar a palavra grega kategoria como um termo técnico para predicação. Os estudiosos dessa tradição acreditam que um sistema de categorias deve ser capaz de fornecer um inventário das coisas que existem. Aristóteles propôs dois 
sistemas de categorias. O segundo, mais conhecido e adotado, contém uma lista de tipos de mais alto nível, os quais são conhecidos como categorias. O fato de que objetos ordinários da experiência humana foram atribuídos a classes de coisas de generalidade crescente, indicando a possibilidade de existência de um único tipo genérico. Entretanto, mesmo Aristóteles não parecia acreditar em um único nível, mas sim em dez, conforme apresentado na Fig.1:

Figura 1 - Categorias genéricas de Aristóteles

\begin{tabular}{|l|l|l|}
\hline \multicolumn{1}{|c|}{ Termo } & \multicolumn{1}{|c|}{$\begin{array}{c}\text { Significado } \\
\text { moderno }\end{array}$} & \\
Aristotélico & \multicolumn{1}{|c|}{ Exemplo } \\
\hline Ti esti, ousia & Substância & homem \\
Poson & Quantidade & cinco metros \\
Poion & Qualidade & branco \\
Pros $t i$ & Relação & no mercado \\
Pou & Local & ontem \\
Pote & Data & sentado \\
Keisthein & Postura & vestido \\
Echein & Estado & queimar \\
Poitein & Ação & ser queimado \\
Paschein & Sentimento & \\
& &
\end{tabular}

Fonte: Adaptado de Sutcliffe (1993)

Para Aristóteles, cada entidade possui uma característica fundamental chamada essência real. Quando uma entidade possui uma essência, ela é de certo tipo; e para ser de certo tipo, a entidade deve compartilhar um conjunto de propriedades necessárias e suficientes com os outros membros daquele tipo (ACKRILL, 1963).

A partir dessa noção nasceu o método aristotélico para distinguir essências que usa a distinção genero-espécie e a divisão dicotômica (LENNOX, 2000). De acordo com a distinção gênero-espécie, a essência real de uma espécie é a combinação de seu gênero e sua differentia, a qual é o critério usado para distinguir uma espécie de outra do mesmo gênero. Por exemplo, a espécie humana pertence ao gênero Homo e sua differentia é a racionalidade. Além disso, através da divisão dicotômica, Aristóteles dividiu cada gênero em entidades que tem certa differentia e naquelas que não tem. Por exemplo, coisas vivas são divididas em animais e plantas pela differentia de auto-movimento.

A tradição aristotélica proporciona assim um método para ordenar categorias de coisas de acordo com suas características essenciais. Kant questionou a maneira não sistemática que as categorias de Aristóteles teriam sido escolhidas e propôs um novo sistema (JANSEN, 2008).

\subsection{Ontologia em Kant}

Para chegar a sua lista de categorias, Kant partiu do conceito de julgamento na forma proposicional do sistema lógico aristotélico, isto é, sujeito-cópula-predicado (por exemplo "S é $P^{\prime \prime}$ ). Quatro aspectos foram propostos para classificar julgamentos: quantidade, qualidade, relação e 
modalidade (WOOD, 2004). Para o aspecto quantidade, o sujeito é de um dos três tipos: universal, particular ou singular.

\begin{tabular}{l|l|l}
\multicolumn{1}{c|}{ Universal } & \multicolumn{1}{c}{ Particular } & \multicolumn{1}{c}{ Singular } \\
\hline Todos S & Algum S & Este S \\
\hline Todos os cães tem pelos & Alguns cães tem pelos & Rex tem pelos
\end{tabular}

Para o aspecto qualidade, aplica-se idéia similar, mas considerando predicação. A diferença entre o julgamento negativo e o infinito é que o primeiro apenas nega um predicado afirmativo, mas o segundo além de negar o predicado afirmativo, deixa em aberto outras possibilidades. Seria correto predicar o número "cinco" como negativo em "cinco não é verde", mas incorreto predicá-lo como infinito, o que significaria que "cinco" não é verde porque tem outra cor.

\begin{tabular}{|c|c|c|}
\hline Afirmativo & Negativo & Infinito \\
\hline $\mathrm{P}$ & não-P & não-P (outros Ps são possíveis) \\
\hline
\end{tabular}

\begin{tabular}{l|l|l}
\hline O livro é vermelho & O livro não é vermelho & O livro não é vermelho, mas ele é azul, amarelo, etc.
\end{tabular}

Para o aspecto modalidade, três tipos de cópula são propostos com o objetivo de conectar sujeito e predicado: problemática, assertória e apodítica

\begin{tabular}{l|l|l}
\multicolumn{1}{c|}{ Problemática } & \multicolumn{1}{c|}{ Assertória } & \multicolumn{1}{c}{ Apoditíca } \\
\hline S é possivelmente P & S é efetivamente P & S é necessariamente P \\
\hline $\begin{array}{l}\text { A mesa é possivelmente } \\
\text { marrom }\end{array}$ & $\begin{array}{l}\text { A mesa é efetivamente } \\
\text { marrom }\end{array}$ & A mesa é necessariamente marrom
\end{tabular}

Finalmente, para o aspecto relação, julgamentos são combinados em inferências silogísticas, como é feito em lógica. Isso resulta em três possibilidades, conforme segue:

\begin{tabular}{l|l|l}
\multicolumn{1}{c|}{ Categórica } & \multicolumn{1}{c}{ Hipotética } & \multicolumn{1}{c}{ Disjuntiva } \\
\hline todo S é P & Se S é P, então S é R & S é P ou R \\
\hline $\begin{array}{l}\text { Todos os animais de } \\
\text { estimação são animais }\end{array}$ & $\begin{array}{l}\text { Se um animal de estimação é um } \\
\text { cão, então ele late }\end{array}$ & $\begin{array}{l}\text { Um cão está latindo ou um gato está } \\
\text { miando }\end{array}$
\end{tabular}

Os aspectos descritos compõem a tabela de julgamentos de Kant (Fig.2, terceira coluna) e a tabela de julgamentos correspondente (FIGURA 2, quarta coluna).

Figura 2 - Tabela de julgamentos e de categorias.

\begin{tabular}{|c|c|c|c|}
\hline & Aspecto considerado & Julgamentos & Categorias \\
\hline \multirow{3}{*}{ I } & \multirow{3}{*}{ Quantidade de julgamentos } & Universal & Unidade \\
\hline & & Particular & Pluralidade \\
\hline & & Singular & Totalidade \\
\hline \multirow{3}{*}{ II } & \multirow{3}{*}{ Qualidade } & Afirmativo & Realidade \\
\hline & & Negativo & Negação \\
\hline & & Infinito & Limitação \\
\hline \multirow{3}{*}{ III } & \multirow{3}{*}{ Relação } & Categórico & Inferência e Subsistência \\
\hline & & Hipotético & Causalidade e Dependência \\
\hline & & Disjuntivo & Reciprocidade \\
\hline \multirow{3}{*}{ IV } & \multirow{3}{*}{ Modalidade } & Problemático & Possibilidade \\
\hline & & Assertório & Existência \\
\hline & & Apodítico & Necessidade \\
\hline
\end{tabular}

Fonte: Adaptado de Kant (1999).

A teoria de Kant lida com categorias do entendimento humano, não com objetos do mundo. Ainda assim, tal teoria mantém importância 
ontológica uma vez que as categorias se aplicam a priori a todos os objetos da cognição.

\subsection{Ontologia em Hursserl}

A fenomenologia desenvolvida por Husserl é basicamente o estudo dos "fenômenos", os quais são as formas nas quais as coisas aparecem para nós em diferentes forma da experiência consciente (SMITH, 2005). Husserl ensina como executar a "redução fenomenológica", se abstendo da percepção do mundo natural e assumindo uma posição transcendental para descrever apenas a consciência pura. O termo "categoria" tem um sentido particular em Husserl: é uma entidade do reino da essências formais, conforme Figura 4 (BEYER, 2011).

$\mathrm{Na}$ ontologia de Husserl, as principais distinções ocorrem entre o reino dos fatos (I), o reino da essências (II) e o reino dos significados ou sentidos (III) (SMITH; SMITH, 2005). Os dois primeiros reinos, fatos e essências, são chamados domínios do conhecimento: o reino dos fatos é o domínio das ciências empíricas tais como física, biologia, psicologia; enquanto o reino da essências é o domínio das ciências eidéticas, tais como matemática, lógica, ontologia formal e material. O terceiro reino, significado, constitui o domínio da fenomenologia. A ontologia de Husserl é apresentada na Figura 3 (SMITH; SMITH, 2005).

Figura 3 - Ontologia básica de Husserl.

- Fatos (entidades concretas)

- Indivíduos

I-1

口 indivíduos independentes (substratos) I-1.1

a indivíduos dependentes (momentos) I-1.2

- Estados das coisas I-2

- Eventos I-3

口 experiências I-3.1

- Essências (entidades ideais) II

- Essência formal II-1

口 Indivíduo II-1.1

- Espécie, Qualidade, Relação II-1.2

- Estado de coisas II-1.3

- Essência material II-2

- Região (Espécie material de mais alto nível) II-3

口 Natureza II-3.1

口 Consciência II-3.2

- Espírito (humanidade) II-3.3

— Significado ou Sentido (conteúdo da experiência) III

- Sentidos dos indivíduos III-1

- Sentidos predicativos de espécies, qualidades e relações III-2

- Proposições

III-3

Fonte: Adaptado de Smith e Smith (2005)

O reino dos fatos (I) inclui entidades concretas, as quais são indivíduos empíricos concretos, estados de coisas empíricos concretos e eventos empíricos concretos. Nesse contexto, "concreto" refere-se à entidades reais que existem no tempo e espaço; e "empírico" refere-se à 
entidades estudadas pelas ciências empíricas, em oposição com aquelas estudadas pela fenomenologia de Husserl. Indivíduos (I-1) podem ser indivíduos independentes (I-1.1), chamados substratos; ou indivíduos dependentes (I-1.2), chamados momentos. Exemplos de substratos são árvores e mesas; exemplos de momentos são instâncias de cores de árvores. Um estado de coisas (I-2) é categoricamente formado por indivíduos. Exemplos de tal configuração são uma mesa e sua cor marrom, uma árvore e sua cor verde, etc. Eventos (I-3) são entidades que tem duração. Um evento ocorre para um indivíduo em certo instante de tempo. Os tipos de eventos mais importantes são as experiências.

O reino das essências (II), ao contrário do reino dos fatos, contém apenas entidades ideais. Entidades ideais são aquelas não-reais, ou seja, não existem no espaço e no tempo. No escopo do reino das essências existem as essências formais (II-1) e as essências materiais (II-2), além das regiões (II-3). Essências formais (II-1) contém entidades que são formas puras representativas de espécies materiais. Essas formas puras são categorias. Dentre as mais altas categorias, aquelas que definem as formas de todos os objetos do mundo são: indivíduos (II-1.1), essências inclusive espécies, qualidades e relações (II-1.2) - e estados de coisas (II-1.3). Essências formais são o conteúdo das ontologias formais. Essências materiais (II-2) consistem de hierarquias de entidades das ciências naturais, organizadas em um formato gênero-espécie. Essências materiais são conteúdo de ontologias materiais. Elas são distintas em natureza (II.3-1), as quais incluem todas as coisas naturais, tais como animais e plantas; consciência, que inclui todas as experiências conscientes; e espírito (II-3.3), que inclui percepção, julgamento e imaginação.

O reino do significado ou sentido (III) contém os significados, isto é, como as pessoas pensam os objetos e os tipos de objetos. Significados não se localizam no tempo e espaço, eles são ideais, mas não são essências; são o conteúdo de experiências intencionais, as formas nas quais objetos são apresentados na consciência. Uma experiência intencional tem conteúdo, a parte central do que é chamado sentido (III1).

\section{Ontologia além da Filosofia}

Além de seu uso em Filosofia, o termo ontologia tem sido empregado em outros campos de pesquisa para se referir a coisas diferentes. Esta seção apresenta uma breve descrição das principais abordagens relativas a ontologias e Ontologia, tanto em Ciência da Computação quanto em Ciência da Informação.

\subsection{Ontologias em Ciência da Computação}

Em Representação do Conhecimento (RC), um sub-campo da Inteligência Artificial (IA), o termo ontologia tem sido usado desde os anos de 1960 para se referir a uma estrutura de conceitos representados 
por um vocabulário lógico. Nos anos de 1990, o termo manteve sua presença no contexto do conjunto de tecnologias que se convencionou rotular de "Web Semântica".

Considerando as atividades e os agentes envolvidos na tarefa de representar conhecimento, é possível melhor entender o papel da ontologia em RC. Sistemas declarativos contém declarações que representam fatos governados por regras. Um exemplo de um fato é "New York é uma cidade nos Estados Unidos" e uma regra é "todas as pessoas que vivem em New York vivem nos Estados Unidos". Essa combinação de fatos e regras compõem uma base de conhecimento do sistema. Uma base de conhecimento é construída e mantida por um engenheiro do conhecimento, que tem como tarefa formalizar o conhecimento de um grupo de especialistas.

Para executar tais tarefas, um engenheiro realiza generalizações e abstrações, as quais requerem insights metafísicos (relacionados à Ontologia). Nessa linha de pensamento, uma ontologia é uma teoria representativa dos principais fatos e regras que governam parte da realidade, com fins computacionais. O termo ontologia, assim, é assim outro nome para essa teoria que em geral é especificada em lógica.

Existem outras abordagens em RC que empregam linguagens de representação mais expressivas para construir os modelos da teoria (GUARINO, 1998). Aderindo a um compromisso ontológico - uma descrição da conceitualização pretendida para uma teoria lógica (GUARINO; GIARETTA, 1995) - emprega-se uma linguagem de representação para gerar um conjunto de modelos representativos da realidade. O papel da ontologia é tornar explícitos axiomas que restringem modelos, de forma a igualar, tanto quanto possível, os modelos que contém o significado pretendido (FIGURA 4).

Figura 4 - Ontologia e linguagem de representação

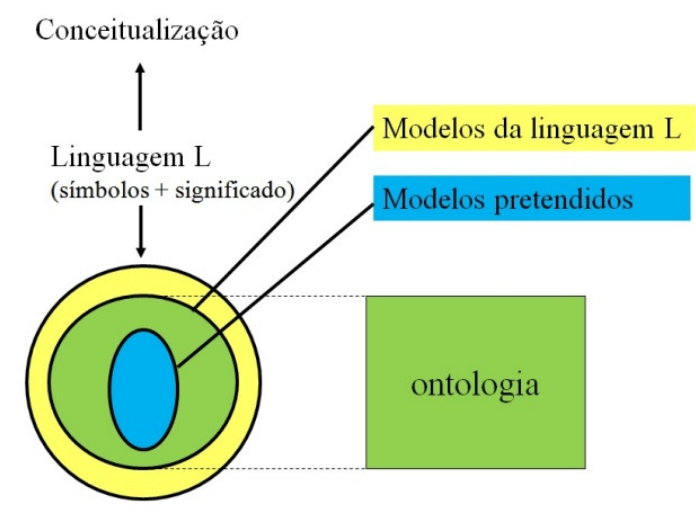

Fonte: Adaptado de Guarino (1998)

Um explicação formal deve considerar uma conceitualização C representada por uma linguagem de representação $L$ que adere a um compromisso ontológico $\mathrm{K}=\langle\mathrm{C}, \mathrm{I}\rangle$. Na verdade, $\mathrm{L}$ se compromete com 0 domínio $D$ através de $K$, do qual $C$ é a conceitualização subjacente. $A$ variável I representa uma função interpretação, a qual mapeia elementos de $\mathrm{D}$ para símbolos do vocabulário $\mathrm{V}$. Todos os modelos de $\mathrm{L}$ que são 
compatíveis com K são modelos pretendidos de $\mathrm{L}$ de acordo com K. Nesse contexto, o papel da ontologia é restringir os modelos de $L$ de forma que eles se tornem modelos pretendidos $I_{k} L$.

Observam-se dois significados principais para o termo ontologia em Ciência da Computação. O primeiro diz respeito ao uso de princípios ontológicos para entender e modelar a realidade (WAND; WEBER, 1990; WAND; STOREY; WEBER, 1999). O uso do termo nesse caso está alinhado com seu papel original na Filosofia, ou seja, fornecer uma descrição do que existe e caracterizar entidades nas atividades de modelagem. $O$ segundo significado diz respeito a representação de um domínio em uma linguagem de representação computacional (STAAB; STUDER, 2004). Uma ontologia, nesse caso, consiste de um conjunto de declarações expressas em um linguagem de representação, o qual pode ser processados por mecanismos de inferência automatizados.

Cabe citar, entretanto, que mesmo considerando o uso de princípios ontológicos no caso da modelagem, nem sempre são tomadas boas decisões Ontológicas para representar a realidade. Decisões são tomadas em função de limitações de desempenho dos sistemas, resultando em representações pobres da realidade (SMITH; WELTY, 2001).

\subsection{Ontologia em Ciência da Informação}

Projetos de engenharia de software envolvendo ontologias exibem paralelos com teorias da Ciência da Informação, tal como classificação facetada, vocabulários controlados e lexicografia (VICKERY, 1997). Existem diferenças e similaridades entre ontologias em Ciência da Computação e outros dois outros termos comuns em Ciência da Informação: taxonomia e tesauros. A possibilidade de restringir a linguagem natural parece ser o ponto de contato entre esses três tipos de estruturas (GILCHRIST, 2003). Além disso, mesmo que o termo ontologia as vezes apareça como um sinônimo para vocabulários controlados, as duas estruturas tem objetivos diferentes (CURRÁS, 2004). Existem ainda outros usos para princípios ontológicos em Ciência da Informação.

Fundamentos filosóficos são utilizados para explicar a hipótese de que a informação tem um status ontológico similar às proposições subjacentes a um texto: ambos são atemporais, não-espaciais e são objetos abstratos (FOX, 1983). Proposições não coincidem com sentenças de um texto, na medida em que várias sentenças podem expressar a mesma proposição. Por exemplo, as sentenças "Marte tem duas luas", "Two moons circle Mars" e "Mars a deux lunes" carregam a mesma proposição, ou seja, o fato de que Marte possui duas luas.

Nessa linha de pensamento, a informação contida em um documento não é identificada com o texto do documento, mas sim como seu conteúdo proposicional. Para alcançar o conteúdo proposicional, é preciso conduzir uma análise proposicional da informação seguindo o seguinte princípio: "a informação transportada pela sentença $\mathrm{S}$ é uma proposição apropriadamente associada a S" (FOX, 1983, p. 84). 
A abordagem proposicional se originou no estudo da Lógica e Filosofia da Linguagem e está relacionada a distinção proposta por Frege. Frege usava como exemplos as expressões "Estrela da manhã" e "Estrela do entardecer", as quais parecem ter diferentes significados, mas se referem a mesma entidade, o planeta Vênus. Frege atribuía as entidades de sua teoria aos três vértices de um triângulo, nomeando-os por símbolo, sentido e referência. Essa mesma distinção foi introduzida na semântica linguística por Ogden e Richards (1972). Dahlberg (1978) apresenta o Triângulo do Conceito no qual o conceito é apresentado como a soma das declarações essenciais e verdadeiras sobre um referente, e o termo (léxico) como a forma comunicável e representável do conceito.

No contexto dos sistemas de recuperação da informação, Blair (2010) torna claro o seu comprometimento com a filosofia (segunda) de Wittgenstein, a qual contém críticas aos princípios ontológicos de Aristóteles. A abordagem de Wittgenstein à categorização é baseada na noção de semelhança de família, a qual tem duas suposições básicas: os membros de um grupo taxonômico compartilham um conjunto de traços similares; tais traços não necessariamente ocorrem em todos os membros de um grupo e apenas naquele grupo (como é o caso na abordagem de Aristóteles).

A significância da visão de Wittgeinstein para o projeto e o uso de sistemas de recuperação da informação reside no fato que "indeterminação será uma característica essencial de muitos esforços de recuperação de conteúdo" (BLAIR, 2010, p. 339). Portanto, deve-se assumir a existência da indeterminação e planejar estratégias para mitigar cada caso, como por exemplo, a sobrecarga de categorias.

Ao que parece, os autores de Ciência da Informação nem sempre mencionam explicitamente o termo "ontologia", mesmo que princípios ontológicos apareçam claramente na literatura daquele campo de pesquisa. Vickery (2007) sugere ainda similaridades com Lancaster (1986), Foskett (1985) e Buckland (1991). Verificar tais pontos de contato está além dos objetivos desse artigo, mas pretende-se que tal iniciativa faça parte de trabalhos futuros.

\section{Discussão}

Apresentaram-se até aqui diferentes usos para o termo "ontologia" em diferentes campos de pesquisa. O levantamento não pretendeu ser exaustivo e pretendeu apresentar noções básicas dos mais influentes sistemas de categorias filosóficos e seu uso em campos da pesquisa aplicada. Além da discussão de aspectos relevantes dos sistemas de categorias, essa seção apresenta uma visão integrada abrangendo as abordagens interdisciplinares.

Mesmo que existam iniciativas para comparar sistemas de categorias filosóficos (MOSS, 1964; PERREAULT, 1994), os sistemas de categorias variam em tal extensão que é difícil acreditar que se possa encontrar um sistema completo e exaustivo (WESTERHOFF, 2005). A despeito desse fato, os sistemas tradicionais continuam a ser empregados 
e novos sistemas têm sido propostos nos últimos 50 anos, a maioria deles baseada naqueles pesquisados nesse artigo. Exemplos desses novos sistemas de fundamentação filosófica são Johansson (1989), Chisholm (1996), Lowe (2007) e Armstrong (1989).

Novos sistemas de categorias também têm sido desenvolvidos também em ciências aplicadas para atender a objetivos específicos já mencionados nesse artigo. Na Ciência da Computação, cabe citar o Descriptive Ontology for Linguistic and Cognitive Engineering (DOLCE) (GANGEMI et al, 2002) e o Basic Foundational Ontology (BFO) (GRENON; SMITH; GOLDBERG, 2007). Enquanto o primeiro é uma ontologia de alto nível inspirada em Kant, o segundo é uma abordagem realista fundamentada em Husserl e Armstrong. Em Ciência da Informação, sistemas de categorias também tem sido criados, não com o objetivo de representar as coisas do mundo, mas sim o conteúdo de documentos visando recuperação da informação. Um exemplo de sistema de categorias bem conhecido é o trabalho de Ranganathan (1967).

É evidente que os sistemas usados em Ciência da Informação e Ciência da Computação tem diferentes objetivos. A Ciência da Computação usa ontologias para categorizar o mundo, mas enfatiza o processo de inferência. Ao contrário, a preocupação da Ciência da Informação não é a inferência lógica, mas sim a manipulação de documentos que descrevem entidades do mundo no âmbito de diferentes domínios de conhecimento e a partir de diferentes pontos de vista.

Considerando as distinções apresentadas até aqui, o Quadro 1 resume as principais interpretações de ontologias em diferentes contextos.

Quadro 1 - Quadro sinótico resumindo as visões sobre Ontologia e ontologias

\begin{tabular}{|c|c|c|c|c|}
\hline Distinção & Campo & O que é? & Propósito & Exemplo \\
\hline $\begin{array}{l}\text { Ontologia } \\
\text { como uma } \\
\text { disciplina }\end{array}$ & Filosofia & $\begin{array}{l}\text { Ontologia como um } \\
\text { sistema de categorias }\end{array}$ & $\begin{array}{l}\text { Entender a realidade, as coisas } \\
\text { que existem e suas características }\end{array}$ & $\begin{array}{l}\text { Sistemas de } \\
\text { Aristóteles, Kant, } \\
\text { Husserl }\end{array}$ \\
\hline \multirow{4}{*}{$\begin{array}{l}\text { Ontologia } \\
\text { como um } \\
\text { artefato }\end{array}$} & \multirow{2}{*}{$\begin{array}{l}\text { Ciência da } \\
\text { Computação }\end{array}$} & $\begin{array}{l}\text { ontologia como uma } \\
\text { teoria (baseada em } \\
\text { lógica) }\end{array}$ & $\begin{array}{l}\text { Entender um domínio e reduzi-lo } \\
\text { à modelos }\end{array}$ & $\begin{array}{l}\text { BFO, DOLCE } \\
\text { (genéricas) }\end{array}$ \\
\hline & & $\begin{array}{l}\text { ontologia como um } \\
\text { artefato de software }\end{array}$ & $\begin{array}{l}\text { Criar um vocabulário para } \\
\text { representação em sistemas e para } \\
\text { gerar inferências }\end{array}$ & $\begin{array}{l}\text { OWL (linguagem } \\
\text { de RC) }\end{array}$ \\
\hline & \multirow{2}{*}{$\begin{array}{l}\text { Ciência da } \\
\text { Informação }\end{array}$} & $\begin{array}{l}\text { ontologia como uma } \\
\text { teoria (informal) }\end{array}$ & $\begin{array}{l}\text { Entender um domínio e } \\
\text { classificar termos }\end{array}$ & $\begin{array}{l}\text { Sistema de } \\
\text { classificação de } \\
\text { Ranganathan }\end{array}$ \\
\hline & & $\begin{array}{l}\text { ontologia como um } \\
\text { sistema conceitual } \\
\text { informal }\end{array}$ & $\begin{array}{l}\text { Criar vocabulários controlados } \\
\text { para recuperação da informação a } \\
\text { partir de documentos }\end{array}$ & $\begin{array}{l}\text { um catálogo, um } \\
\text { glossário, um } \\
\text { tesauros }\end{array}$ \\
\hline
\end{tabular}

Fonte: Desenvolvido pelo autor

A última linha do Quadro 1 classifica um catálogo, um glossário e um tesauro como um ontologia, de acordo com a visão de Smith e Welty (2001), a qual considera todas essas estruturas como sistemas 
conceituais informais. Cabe destacar que essa visão é possível mas discutível: uma ontologia pode não ser considerada um sistema de classificação ou um vocabulário controlado no sentido que esses termos são utilizados em Ciência da Informação, ou seja, como um conjunto normalizado e autorizado de termos de indexação (JACOB, 2005).

\section{Conclusões}

No presente artigo, diferentes perspectivas relacionadas ao termo "ontologia" foram revisitadas, destacando-se a Filosofia como campo original de pesquisa. Três influentes sistemas no escopo da Ontologia foram descritos - um realista, um cognitivo e um descritivo - os quais correspondem, respectivamente, a teorias desenvolvidas por Aristóteles, Kant e Husserl. Em seguida, apresentou-se uma visão geral do uso do termo "ontologia" além da Filosofia, destacando como o termo tem sido empregado em outros campos de pesquisa aplicados. Explicou-se o interrelacionamento entre esses campos de pesquisa e discutiu-se algumas questões relacionadas a ontologias. Nesse momento, cabe oferecer conclusões e explicar oportunidades de pesquisa interdisciplinar.

O termo ontologia em Ciência da Computação é usado para se referir tanto a um vocabulário expresso em uma linguagem de RC, quanto a um tipo de teoria onde fenômenos são explicados a partir de fatos e regras. O primeiro uso corresponde a um software, um artefato computacional. O segundo uso mantém a noção filosófica, um inventário de coisas do mundo e relações entre elas em um domínio particular, baseado nos princípios da Ontologia como disciplina.

Em Ciência da Informação, princípios ontológicos são usados no suporte à construção de estruturas de categorização para representação do conteúdo de documentos. De fato, o trabalho em ontologias na Ciência da Informação não está confinado a representação de conteúdo, mas engloba a representação desses recursos como um todo, em geral, da perspectiva de uma comunidade específica de usuários. Ontologia, nesse sentido, é um assunto frutífero para pesquisa em Ciência da Informação, uma vez que mantém o potencial para explicar também todo o ambiente social envolvido na análise conduzida pelo cientista da informação. Os interessados nessa possibilidade podem pesquisar a ontologia do social em Zaibert e Smith (2010), Tsohatzidis (2007), Tuomela (2007), Gilbert (1989), Mulligan (1987).

Evidências do uso de princípios ontológicos em Ciência da Informação foram apresentados ao longo do artigo. Questões complexas e relevantes - por exemplo, a busca de uma definição para informação foram abordadas a partir de uma perspectiva Ontológica. Mesmo considerando-se as ontologias computacionais que têm sido criadas em grande escala em domínios como a Medicina (SMITH et al, 2007), essa iniciativas fazem uso de sistemas de categorias, os mesmos utilizados em Ciência da Informação. É possível interpretar as iniciativas de ontologias computacionais como grandes repositórios de conhecimento científico, os 
quais, de outra perspectiva, são objeto de interesse da Ciência da Informação.

O que de fato conecta os diferentes campos de pesquisa mencionados ao longo do artigo é o que tem sido chamado aqui de "princípios ontológicos". Eles quais são o elemento comum presente nas iniciativas da Filosofia, Ciência da Computação e Ciência da Informação. De forma a alcançar um nível interdisciplinar de pesquisa, a tarefa de manipular os princípios ontológicos deve ocorrer em dois estágios: 0 primeiro é a - priori e devotado a estabelecer que tipos de coisas podem existir e co-existir no mundo; o segundo envolve um esforço para estabelecer que tipos de coisas existem de acordo com evidências empíricas. A primeira tarefa tem lugar na Filosofia, a segunda nas ciências aplicadas, como por exemplo, a Ciência da Informação.

O benefício para a Filosofia é o teste sistemático de princípios Ontológicos em empreendimentos práticos, fornecendo um novo uso para as noções teóricas filosóficas em importantes campos como Medicina ou Direito. O benefício para as Ciências Aplicadas é ter a disposição princípios sólidos e robustos, alguns dos quais em discussão por mais de dois mil anos, para fundamentar investigações empíricas. No âmbito desse cenário residem as oportunidades para pesquisa interdisciplinar em ontologias. Nesse contexto, a literatura de Ciência da Informação sobre ontologias deve ser construída.

\section{Referências}

ACKRILL, J. L. Aristotle: Categories and De Interpretatione. Oxford: Clarendon, 1963.

ARMSTRONG, D. M. Universals: An Opinionated Introduction. London: Westview, 1989.

AYERS, M. R. Locke Versus Aristotle on Natural Kinds.1981. Disponível em: <http://filebox.vt.edu/users/ottw/ayers\%20natural\%20kinds.pdf>. Acesso em: 5 dez. 2011.

BEYER, C. Edmund Husserl. 2011. Disponível em: <http://plato.stanford.edu/entries/husserl/>. Acesso em: 5 fev. 2012.

BLAIR, D. C. Wittgenstein, language and information: back to the rough ground. Amsterdan: Springer, 2006.

BUCKLAND, M. K. Information as Thing. 1991. Disponível em: <http://www.publicpraxis.com/wpcontent/uploads/2011/01/informationasthing.pdf $>$. Acesso em: $10 \mathrm{fev}$. 2012.

CAMPOS, M. L. A.; CAMPOS, L.; MEDEIROS, J. S. A Representação de Domínios de Conhecimento e uma Teoria de Representação: a ontologia de fundamentação. 2011. Disponível em: <http://www.uel.br/revistas/uel/index.php/informacao/article/view/10389 >. Acesso em: 10 jan. 2013 
CHISHOLM, R. A realistic theory of categories. Cambridge: Cambridge Press, 1996.

CURRÁS, E. Ontologies, Taxonomies and Thesauri in Systems Science and Systematics. Cambridge: Woodhead, 2003.

COHEN, S. M. Aristotle's Metaphysics. 2008. Disponível em: $<$ http://plato.stanford.edu/entries/aristotle-metaphysics/>. Acesso em: 10 fev. 2012.

FONSECA, F. The Double Role of Ontologies in Information Science Research. Journal of the American Society for Information Science and Technology, Maryland, v.58, n.6, p.786-793, 2007.

FOSKETT, D. J. Classification and integrative levels. In: CHAN, L.M. et al. (Ed.). Theory of Subject Analysis. Littleton: Libraries Unlimited, 1985. p. 212-220.

FOX, C. J. Information and Misinformation: An Investigation of the Notions of Information, Misinformation, Informing, and Misinforming. Westport: Greenwood, 1983.

GANGEMI, A.; GUARINO, N.; MASOLO, C.; OLTRAMARI, A.; OLTRAMARI, R.; SCHNEIDER, L. (2002). Sweetening Ontologies with DOLCE. (2002). Disponível em: <http://www.loa.istc.cnr.it/Papers/DOLCE-EKAW.pdf>. Acesso em 10 jan. 2010.

GILBERT, M. On Social Facts. London: Routledge, 1989.

GILCHRIST, A. Thesauri, Taxonomies and Ontologies; an etymological note. 2003.2 Disponível em: <http://dois.mimas.ac.uk/DoIS/data/Articles/julkokltny:2003:v:59:i:1:p:

7-18.html>. Acesso em: 2 mar. 2009.

GRENON, P.; SMITH, B.; GOLDBERG, L. Biodynamic Ontology: Applying $\mathrm{BFO}$ in the Biomedical Domain. 2007. Disponível em: <http://ontology.buffalo.edu/medo/biodynamic.pdf>. Disponível em: 20 set. 2011.

GUARINO, N. Formal Ontology in Information Systems. 1998. Disponível em: <http://citeseer.ist.psu.edu/guarino98formal.html>. Acesso em: 3 mar. 2009.

GUARINO, N.; GIARETTA, P. Ontologies and Knowledge Bases: towards a terminological clarification. $1995 . \quad$ Disponível em: <http://www.loa.istc.cnr.it/Papers/KBKS95.pdf>. Acesso em: 30 jul. 2010.

HANNA, R. Kant, Science, and Human Nature. Oxford: Oxford University Press, 2006.

HUSSERL, E. Logical Investigations. In: MUNN, K.; SMITH, B. (Ed.). Applied Ontology: an Introduction. Berlin: Verlag, 2001. p. 39-56. 
JACOB, E. K. Ontologies and the SemanticWeb. 2005. Disponível em: $<$ http://onlinelibrary.wiley.com/doi/10.1002/bult.283/abstract>. Acesso em: 20 jan. 2013.

JANSEN, L. Categories: The Top Level Ontology. In: MUNN, K.; SMITH, B. (Ed.). Applied Ontology: an Introduction. Berlin: Verlag, 2008. p. 173196.

JOHANSSON, I. Ontological Investigations. New York: Routledge, 1989.

KANT, I. Critique of Pure Reason. Cambridge: Cambridge University Press, 1999.

KITCHER, P. A Priori. In: GUYER, P. (Ed.). The Cambridge Companion to Kant and Modern Philosophy. Cambridge: Cambridge Press, 2006. p. 2961.

LENNOX, J. Aristotle's Philosophy of Biology: Studies in the Origins of Life Science. Cambridge: Cambridge University Press, 2000.

LANCASTER, F.W. Vocabulary Control for Information Retrieval. Arlington: Information Resources Press, 1986.

LOWE, E. J. The Four-Category Ontology: A Metaphysical Foundation for Natural Science. New York, NY: Oxford University Press, 2007.

MOSS, R. Categories and Relations: origins of two classification theories. American Documentation, Maryland, v.15, p. 296-301, 1964.

MULLIGAN, K. Speech Act and Sachverhalt: Reinach and the Foundations of Realist Phenomenology. Dordrecht: Nijhoff, 1987.

PERREAULT, J. Categories and relators: a new schema. Knowledge Organization, Würzburg, v.21, n. 4, p. 189-198, 1994.

RANGANATHAN, S. R. Prolegomena to Library Classification. London: Asia Publishing House, 1967.

RITTGEN, P. Handbook of Ontologies for Business Interaction. Hershey: IGI Global, 2012.

ROHLF, M. Immanuel Kant. 2010. Disponível em: <http://plato.stanford.edu/entries/kant/>. Acesso em: 23 out. 2009.

SCHIESSL, M.; BRÄSCHER, M. Do texto às ontologias: uma perspectiva para a ciência da informação. 2011. Disponível em: <http://revista.ibict.br/cienciadainformacao/index.php/ciinf/article/view/1 947>. Acesso em: 10 fev. 2013.

SMITH, B.; SMITH, D.W. The Cambrigde Companion to Husserl. Cambridge: Cambridge Press, 2005.

SMITH, D. W. Phenomenology. 2003. Disponível em: $<$ http://plato.stanford.edu/entries/phenomenology/>. Acesso em: 3 jul.2010.

SMITH, B. Ontology and Informations Systems. 2004. Disponível em: <http://www.ontology.buffalo.edu/ontology>. Acesso em: 25 jan. 2006. 
SMITH, B.; WELTY, C. Ontology: Towards a new synthesis. 2001. Disponível em: <http://www.cs.vassar.edu/ w weltyc/papers/foisintro.pdf>. Acesso em: 20 dez. 2010.

SMITH, B. et al. The OBO Foundry: coordinated evolution of ontologies to support biomedical data integration. 2007. Disponível em: <http://www.nature.com/nbt/journal/v25/n11/full/nbt1346.html>.

Acesso em: 22 maio 2009.

SMITH, D. W. Mind and Body. In: SMITH, B.; SMITH, D.W. (Ed.). The Cambrigde Companion to Husserl. Cambridge: Cambridge Press, 2005. p. 323-393.

SOERGUEL , D. Functions of a thesaurus, classification and ontological knowledge bases. 1997. Disponível em: <http://www.clis.umd.edu/faculty/soergel/soergelfctclass.pdf>. Acesso em: 11 dez. 2005.

SOERGUEL , D. The rise of ontologies or the reinvention of classification. 1999. Disponível em: <http://www.dsoerguel.com/CV/B70.pdf>. Acesso em: 23 jun. 2008.

STAAB, S.; STUDER, R. Handbook on Ontologies. Berlin: Springer, 2004.

STUDTMANN, P. The foundations of Aristotle's categorial scheme. Milwaukee: Marquette University Press, 2008.

SUTCLIFFE, J. P. Concept, class, and category in the tradition of Aristotle. In: VAN MECHELEN, I.; HAMPTON, J. J.; MICHALSKI, R. S.; THEUNS, P. (Ed.). Categories and Concepts. London: Academic, 1993. p. 35-65.

THOMASON, A. Categories. 2009. Disponível em: <http://plato.stanford.edu/entries/categories/>. Acesso em: 3 mar. 2011.

TSOHATZIDIS, S. L. Intentional Acts and Institutional Facts: Essays on John Searle's Social Ontology. Dordrecht: Springer, 2007.

TUOMELA, R. The importance of us. Palo Alto: Stanford University Press, 2007.

VICKERY, B. C. Ontologies. Journal of Information Science, London, v. 23, n. 4, p. 227-286, 1997.

WAND, Y.; WEBER, R. Mario Bunge's ontology as a formal foundation for information systems concepts. In: WEINGARTNER, P.; DORN, J. W. G. (Ed.). Studies on Mario Bunge's treatise. Amsterdam: Rodopi, 1990. p.123-153.

WAND, Y.; STOREY, V. C.; WEBER, R. An ontological analysis of the relationship construct in conceptual modeling. ACM Transactions on Database Systems, New York, v. 24, n. 4, p. 494-528, 1999.

WESTERHOFF, J. Ontological Categories: Their Nature and Significance. Oxford: Clarendon, 2005. 
WITTGENSTEIN, L. Philosophical Investigations. Chichester: WileyBlackwell, 2009.

WOOD, A. Kant. Oxford: Wiley-Blackwell, 2004.

ZAIBERT, L.; SMITH, B. The Varieties of Normativity: An Essay on Social Ontology. 2010.

em: 18 jan. 2011. 